Article

\title{
A Conserved Female-Specific Requirement for the GGT Gene in Mosquito Larvae Facilitates RNAi-Mediated Sex Separation in Multiple Species of Disease Vector Mosquitoes
}

\author{
Keshava Mysore 1,2 ${ }^{1}$, Longhua Sun ${ }^{1,2}$, Ping Li 1,2, Joseph B. Roethele ${ }^{1,2}$, Joi K. Misenti ${ }^{1,2}$, John Kosmach ${ }^{1,2}$, \\ Jessica Igiede ${ }^{1,2}$ and Molly Duman-Scheel ${ }^{1,2, * \text { (D) }}$ \\ 1 Department of Medical and Molecular Genetics, Indiana University School of Medicine, \\ Raclin-Carmichael Hall, 1234 Notre Dame Ave., South Bend, IN 46617, USA; kmysore@iu.edu (K.M.); \\ Longhua.Sun.15@nd.edu (L.S.); PLi@uams.edu (P.L.); jroethe@iu.edu (J.B.R.); jmisenti@iu.edu (J.K.M.); \\ jkosmach@nd.edu (J.K.); jigiede@nd.edu (J.I.) \\ 2 Eck Institute for Global Health, The University of Notre Dame, Notre Dame, IN 46556, USA \\ * Correspondence: mscheel@nd.edu
}

check for updates

Citation: Mysore, K.; Sun, L.; Li, P.; Roethele, J.B.; Misenti, J.K.; Kosmach, J.; Igiede, J.; Duman-Scheel, M. A

Conserved Female-Specific

Requirement for the GGT Gene in Mosquito Larvae Facilitates RNAi-Mediated Sex Separation in Multiple Species of Disease Vector Mosquitoes. Pathogens 2022, 11, 169. https://doi.org/10.3390/ pathogens11020169

Academic Editors: Shengzhang Dong and Eric Caragata

Received: 9 December 2021

Accepted: 26 January 2022

Published: 27 January 2022

Publisher's Note: MDPI stays neutral with regard to jurisdictional claims in published maps and institutional affiliations.

Copyright: (C) 2022 by the authors. Licensee MDPI, Basel, Switzerland. This article is an open access article distributed under the terms and conditions of the Creative Commons Attribution (CC BY) license (https:// creativecommons.org/licenses/by/ $4.0 /)$.

\begin{abstract}
Although several emerging mosquito control technologies are dependent on mass releases of adult males, methods of sex-sorting that can be implemented globally have not yet been established. RNAi screens led to the discovery of siRNA, which targets gamma-glutamyl transpeptidase (GGT), a gene which is well conserved in multiple species of mosquitoes and located at the sex-determining $\mathrm{M}$ locus region in Aedes aegypti. Silencing the A. aegypti, Aedes albopictus, Anopheles gambiae, Culex pipiens, and Culex quinquefasciatus GGT genes resulted in female larval death, with no significant impact on male survival. Generation of yeast strains that permitted affordable expression and oral delivery of shRNA corresponding to mosquito GGT genes facilitated larval target gene silencing and generated significantly increased 5 males: 1 female adult ratios in each species. Yeast targeting a conserved sequence in Culex GGT genes was incorporated into a larval mass-rearing diet, permitting the generation of fit adult male C. pipiens and C. quinquefasciatus, two species for which labor-intensive manual sex separation had previously been utilized. The results of this study indicate that femalespecific yeast-based RNAi larvicides may facilitate global implementation of population-based control strategies that require releases of sterile or genetically modified adult males, and that yeast RNAi strategies can be utilized in various species of mosquitoes that have progressed to different stages of sex chromosome evolution.
\end{abstract}

Keywords: Aedes aegypti; Aedes albopictus; Anopheles gambiae; Culex pipiens; Culex quinquefasciatus; development; female lethal; gamma-glutamyl transpeptidase; larvicide; male; RNAi; Saccharomyces cerevisiae; sex; yeast

\section{Introduction}

Mosquito-borne diseases lead to hundreds of thousands of human deaths annually [1]. Although mosquito control is critical for prevention of mosquito-borne illnesses, the emergence of insecticide resistance, concern for the unwanted negative effects of insecticides on non-target organisms, and a general lack of support for mosquito control programs threaten current schemes for managing mosquitoes worldwide [2]. These problems have led to increased interest in alternative control methods, such as the sterile insect technique (SIT), which was proposed decades ago [3] and entails the release of sterile adult males with the goal of reducing large populations of insects. The broad implementation of SIT for control of mosquito populations has been hampered by a lack of cost-effective and scalable sex-sorting technologies that can be adapted for use worldwide in multiple mosquito species [4,5]. The use of males is critical for minimizing the health and nuisance biting risks posed by the release of females [4,5]. Likewise, the incompatible insect technique (IIT), which requires 
the release of Wolbachia-infected adult males for mosquito population suppression, can be paired with SIT and also involves mass-rearing and sex separation [6-8]. Transgenic-based population suppression approaches, including the release of insects which carry a dominant lethal (RIDL) $[9,10]$, as well as several emerging gene drive technologies, also involve male mosquito releases [11,12]. Unfortunately, visual and mechanical separation technologies, the only existing sex-sorting methods for some species of mosquitoes, including Culex pipiens complex mosquitoes, are highly labor intensive and not sufficient for the large-scale implementation of SIT or IIT programs [5]. The establishment of sex separation methods is thus a rate-limiting step in the global deployment of several emerging population-based mosquito control technologies [5].

The identification and characterization of genes with sex-specific functions could promote the elucidation of male sex-sorting technologies to support emerging mosquito control interventions. In A. aegypti, sex determination is regulated by a non-recombining Y-chromosome-like male-determining region, referred to as the $\mathrm{M}$ locus, which is present on chromosome one [13,14] and contains the male-determining factor Nix [15]. A. aegypti males, which have one copy of the chromosome bearing the $\mathrm{M}$ locus and one which lacks it, have an $\mathrm{M} / \mathrm{m}$ genotype. A. aegypti females, which lack the male-determining locus, have an $\mathrm{m} / \mathrm{m}$ genotype [16]. Characterization and sequencing of the $\mathrm{M} / \mathrm{m}$ locus had been obstructed by the presence of highly repetitive DNA at this centromeric region of chromosome one. However, recent innovations in sequencing technology generated an improved and re-annotated genome assembly $[17,18]$ that enabled better estimation of the $\mathrm{M} / \mathrm{m}$ locus. These efforts revealed the presence of many long non-coding RNA (IncRNA) genes, as well as several protein-encoding genes that are located at or tightly linked to the $\mathrm{M} / \mathrm{m}$ locus [17], a location that is referred to herein as the $\mathrm{M} / \mathrm{m}$ locus region.

Although clusters of loci that cause sex-specific lethal effects are proposed to reside within the $\mathrm{M} / \mathrm{m}$ region $[19,20]$, the identities of these genes were previously unknown. A recent RNA interference (RNAi) screen led to the identification of female-specific larval lethal genes located in the $A$. aegypti sex-determining region, including multiple $\ln C R A$

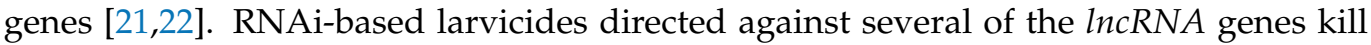
female larvae during development; however, they have no impact on male mosquitoes [21]. Unfortunately, $\operatorname{lnc} R N A$ genes have not yet been annotated in most mosquito species, and orthologs for these $\mathrm{M} / \mathrm{m}$ locus region genes have not been described in other mosquitoes [21]. However, recent characterization of $A$. aegypti $M t n B$, a protein-encoding gene located in the $\mathrm{M}$ locus region that has known orthologs in other dipterans, indicated that a female-specific larval requirement of this gene is well-conserved among mosquitoes [22]. These results, which are interesting given that a sex-determining region on homomorphic chromosome 1 controls sex in Aedes and Culex mosquitoes, whereas A. gambiae has evolved heteromorphic $X$ and $Y$ chromosomes [23], support the hypothesis that a female-specific requirement for protein-encoding genes located at the A. aegypti $\mathrm{M} / \mathrm{m}$ locus region is conserved among mosquitoes. Further evaluation of this hypothesis through examining the function of other $\mathrm{M} / \mathrm{m}$ locus region genes is expected to provide insight into the evolution of mosquito sex chromosomes and sex-specific development.

Here, we pursue RNAi-mediated silencing of gamma-glutamyl transpeptidase (GGT), a gene located in the $\mathrm{M} / \mathrm{m}$ locus region of $A$. aegypti. GGT is conserved in multiple species of dipteran insects, including disease vector mosquitoes. In Drosophila melanogaster, GGT, which is located on the $X$ chromosome, is predicted to have glutathione hydrolase activity and peptidyltransferase activity [24]. GGT is expressed in a variety of D. melanogaster tissues, including the adult head, the embryonic and larval circulatory system, and midline ventral glial cells, in which it is proposed to function in the glutathione catabolic processes [24]. The human GGT gene has been implicated in glutathionuria, a disorder that is characterized by increased glutathione concentration in the plasma and urine [25]. Based on recent analyses of other $\mathrm{M} / \mathrm{m}$ locus region genes in $A$. aegypti [21,22], it was hypothesized that silencing GGT during mosquito larval development will result in significantly higher male:female sex 
ratios in multiple mosquito species, and that the development of scaled RNAi approaches for targeting GGT could facilitate the mass-rearing of male mosquitoes.

Saccharomyces cerevisiae, which is genetically tractable and inexpensive to produce at scale, can be engineered to produce interfering RNA that silences mosquito genes upon consumption of the yeast by larvae [26-28]. Yeast RNAi strains promote effective gene silencing during the larval stages, and yeast RNAi larvicides generate relatively higher levels of larval mortality than soaking or chitosan RNAi larvicide delivery methods do [26,27]. Moreover, RNA can be generated through yeast culturing, significantly reducing interfering RNA production costs [28]. The interfering RNA also retains activity when the yeast is heat-killed and dried, which allows it to be more readily packaged, shipped, stored, and used in an inactivated form [26,27]. Initial studies in A. aegypti suggest that yeast RNAi silencing of $\mathrm{M} / \mathrm{m}$ locus region genes permits the scaled production of fit adult males [21,22]. Here, we extend the use of this yeast system to the characterization of mosquito GGT orthologs, as well as to the scaled production of male Culex pipiens complex mosquitoes.

\section{Results}

\subsection{RNAi Assays Identify GGT as a Female-Specific Larval Lethal Gene in A. aegypti}

Given evidence that the A. aegypti $\mathrm{M} / \mathrm{m}$ locus is tightly linked to developmental genes that confer sex-specific effects $[21,22,29]$, it was hypothesized that the A. aegypti GGT gene, which is located on Chromosome 1 and flanks the sex determination $\mathrm{M} / \mathrm{m}$ locus $[17,18]$, functions as a sex-specific lethal gene. In support of this hypothesis, larval soaking assays performed with siRNA \#546, which corresponds to the GGT transcript, induced significant larval mortality, resulting in $25 \pm 3 \%$ of expected adult females $(p<0.001)$, but had no significant impact on male survival. Based on the outcome of these trials, yeast strain GGT.546, which expresses an shRNA corresponding to the siRNA \#546 target site in GGT, was constructed and found to induce $94 \pm 1 \%$ reduction in GGT transcript levels during larval development (Figure 1A, $p<0.001$ in comparison with larvae reared on control-interfering RNA yeast, which expresses an shRNA hairpin with no known target in mosquitoes, and which is referred to as control-interfering RNA yeast hereafter). In small container trials performed on 20 larvae, treatments with GGT.546 throughout larval development induced significant larval death (Figure 1B, $p<0.001$ ), yielding only $22 \pm 4 \%$ of expected females, yet had no significant impact on male survival (Figure 1B, $p>0.05$ ), resulting in significantly higher male:female ratios among surviving adult mosquitoes (Figure $1 \mathrm{~B}, p<0.001$ ). Larval death occurred primarily during the third larval instar, as shown in the survival curve in Figure 1C.

GGT.546 larvicide was also evaluated when 500 larvae were reared in larger rearing trays. In these scaled rearing experiments, GGT.546 yeast was incorporated into a larval mass-rearing diet (Figure 1E). Significant larval mortality resulted when larvae consumed this diet, yielding $15 \pm 1$ of expected adult female survival. There was no significant impact on male survival (Figure 1F) or wing length, a correlate of fitness (though fitness was not measured directly, Figure 1D), resulting in significantly higher male:female ratios among surviving adult mosquitoes (Figure 1E, $p<0.001$ ).

\subsection{The Female-Specific Larval Requirement for GGT Is Conserved in Multiple Species of Disease Vector Mosquitoes}

GGT orthologs have been identified in the genomes of A. albopictus, C. pipiens, C. quinquefasciatus, and A. gambiae (Table 1). siRNAs \#565 (which corresponds to A. albopictus GGT), \#560 (which targets A. gambiae GGT), and \#566 (which corresponds to both the C. quinquefasciatus and C. pipiens GGT genes) induced significant female larval death, yet did not affect male survival following soaking treatments of each respective species (detailed results and statistics are provided in Table 1). Based on these results, as well as those observed in $A$. aegypti (Figure 1, Table 1), yeast strains which express shRNA corresponding to either the 
\#560, \#565, or \#566 target sites, hereafter referred to as GGT.560, GGT.565, and GGT.566, were thus constructed. shRNA expression was verified in each of these strains (Figure S1).
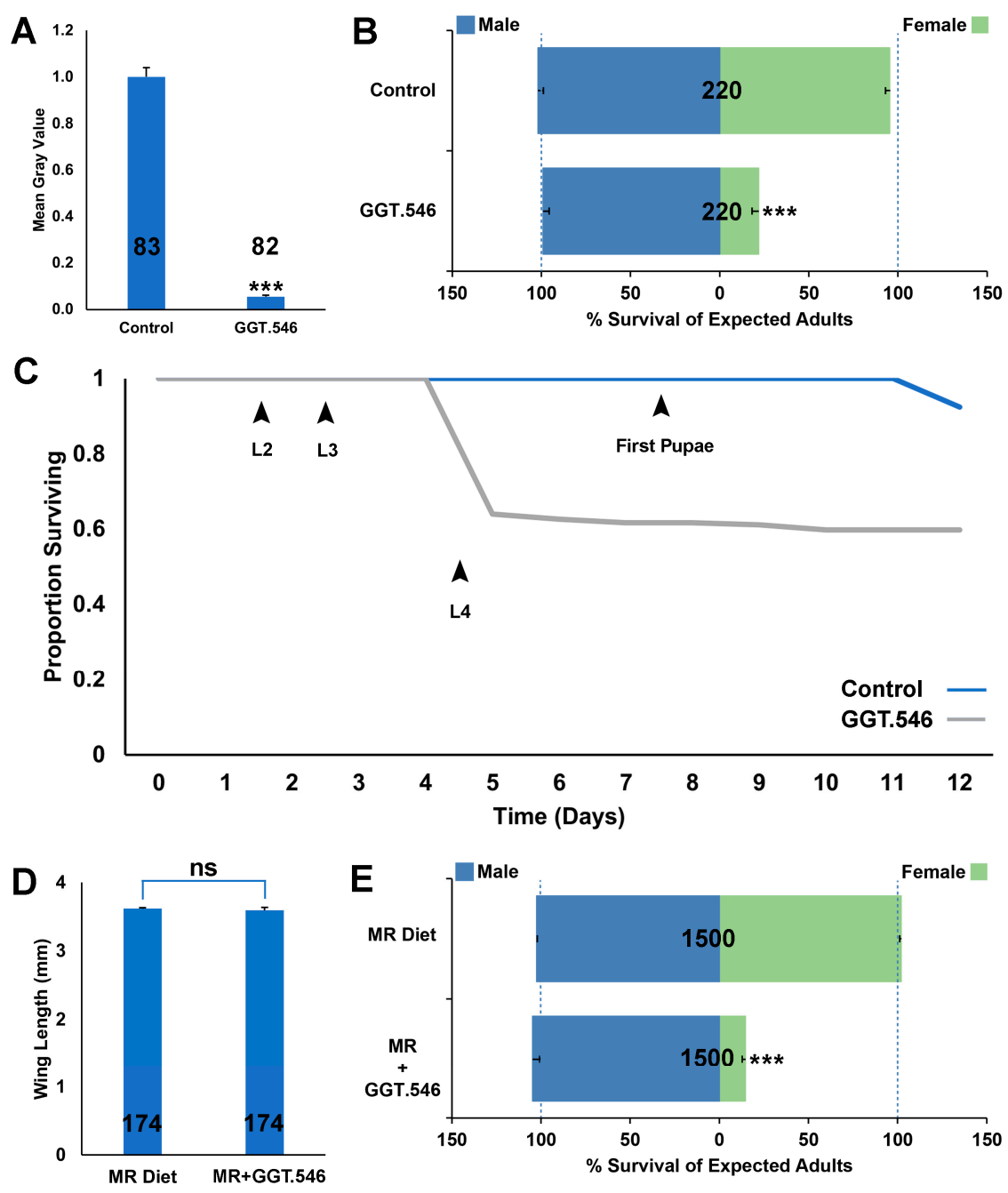

Figure 1. Yeast larvicide GGT.546 functions as a female-specific larvicide in Aedes aegypti. (A) Silencing of GGT in A. aegypti following larval consumption of GGT.546 yeast was verified through mean grey value quantification of GGT transcripts following in situ hybridization experiments performed on L4 brain tissue ${ }^{* * *}=p<0.001$, Student's $t$-test; error bars denote standard deviation). Larval consumption of GGT.546 yeast resulted in significant female larval mortality (B), which occurred primarily in the third instar (GGT.546-treated larvae = gray line in survival curve shown in (C); compared with the blue line, which denotes the survival of control-interfering RNA yeast-treated larvae), yet did not significantly impact survival of males through adulthood ( $p>0.05$, chi-squared test). (D) No significant differences in mean wing lengths were detected in adult males reared following scaled rearing of larvae on a mass-rearing diet prepared with GGT.546 yeast (MR+GGT.546; $p>0.05$ vs. wings from males reared on the standard mass-rearing diet (MR Diet), which served as a control, $t$-test). Although the consumption of MR+GGT.546 resulted in significant female mortality, it had no impact on male survival (E). The results shown in panels $(\mathbf{B}, \mathbf{C})$ correspond to container trials performed on 20 larvae per replicate container; results from larvae that had consumed controlinterfering RNA yeast, which had no impact on male or female survival ( $p>0.05$, chi-squared test) are shown for comparison. Results shown in $(\mathbf{D}, \mathbf{E})$ correspond to trials performed in scaled rearing trays containing 500 larvae that consumed the MR diet or the MR+GGT.546 diet; data are shown as mean survival through adulthood; ${ }^{* *}=p<0.001$, chi-squared test. Throughout this figure, the $\mathrm{N}$ numbers for each treatment are indicated on the respective columns of each bar graph, and error bars represent standard errors of the mean. 
Table 1. Female-specific siRNA target sequences/genes and male:female sex ratios resulting from treatments.

\begin{tabular}{|c|c|c|c|c|c|c|}
\hline siRNA & Target Sequence & Corresponding Genes & Species & $\begin{array}{l}\text { siRNA Soaking } \\
\text { Treatment } \\
\text { \#Males:\#Females } \\
\text { (N) }\end{array}$ & $\begin{array}{c}\text { Male } \\
\text { Mortality } \\
p \text { Value }\end{array}$ & $\begin{array}{c}\text { Female Mortality } \\
p \text { Value }\end{array}$ \\
\hline Control & GAAGAGCACUGAUAGAUGUUAGCGU & N/A & A. aegypti & $20: 20(40)$ & 1.00 & 1.00 \\
\hline $546^{1}$ & GAAGCAUUCAAACAUGCUUACGGCA & AAEL017331 & A. aegypti & $23: 5(40)$ & 1.00 & $3.85 \times 10^{-7}$ \\
\hline Control & GAAGAGCACUGAUAGAUGUUAGCGU & N/A & A. albopictus & $20: 19(40)$ & 1.00 & 1.00 \\
\hline 565 & GCAUCAAGCUUGAUGAUGAAAUUUA & LOC109416314 & A. albopictus & $22: 5(40)$ & 0.48 & $4.93 \times 10^{-6}$ \\
\hline Control & GAAGAGCACUGAUAGAUGUUAGCGU & N/A & C. quinquefasciatus & 19:21(40) & 1.00 & 1.00 \\
\hline 566 & AGACUUACGAUUGAUGAAAACAGUA & CPIJO16229 & $\begin{array}{l}\text { C. quinquefasciatus } \\
\text {. }\end{array}$ & $19: 4(40)$ & 0.50 & $7.7 \times 10^{-8}$ \\
\hline Control & GAAGAGCACUGAUAGAUGUUAGCGU & N/A & A. gambiae & $19: 18(40)$ & 1.00 & 1.00 \\
\hline 560 & CUAUCUGUUUGACCAGUUUACCGTC & AGAP000853 & A. gambiae & $19: 3(40)$ & 0.25 & $1.28 \times 10^{-8}$ \\
\hline
\end{tabular}

${ }^{1}$ siRNAs and corresponding target sequences/genes in the indicated species and the altered adult surviving male:female ratios resulting from siRNA soaking treatments are indicated. The $p$ values displayed correspond to Fisher's exact tests that compared observed and expected male or female survival (assuming 20 female: 20 male adult survivors, as predicted based on experimentation with these strains in our laboratory).

Rearing A. albopictus larvae on GGT.566 yeast resulted in significantly higher than expected male:female ratios (Figure $2 \mathrm{~A}, p<0.001$ ). Although only $22 \pm 3 \%$ of expected $A$. albopictus female mosquitoes treated with GGT.566 emerged as adults (Figure 2A, $p<0.001$ ), no significant impact on male survival was observed (Figure 2A, $p>0.05$ ). Likewise, although GGT.560 had no significant impact on the survival of males through adulthood (Figure 2B, $p>0.05$ ), only $22 \pm 2 \%$ of expected $A$. gambiae female mosquitoes survived through adulthood (Figure $2 \mathrm{~B}, p<0.001$ ), resulting in a significantly higher male:female ratio among adult survivors (Figure $2 \mathrm{~B}, p<0.001$ ). Finally, significantly higher than expected male:female ratios were also observed among both C. pipiens (Figure $2 \mathrm{C}, p<0.001$ ), and C. quinquefasciatus (Figure 2D, $p<0.001$ ) adult survivors following larval rearing on GGT.566 yeast, a difference that coincided with $22 \pm 3 \%$ of expected C. pipiens (Figure 2C, $p<0.001$ ), and $22 \pm 3 \%$ of expected C. quinquefasciatus (Figure 2D, $p<0.001$ ) female adult emergence, yet had no impact on male survival of either species (Figure $2 \mathrm{C}, \mathrm{D}, p>0.05$ ).

\subsection{The Use of GGT.566 Yeast in Scaled Production of Adult C. pipiens Complex Male Mosquitoes}

To assess whether the RNAi-based yeast larvicides could also facilitate scaled production of $C$. pipiens complex male mosquitoes, a larval diet and feeding regimen employed at Aedes mass-rearing facilities [30] was modified through replacement of the nutritional yeast component of the diet with dried inactivated yeast larvicide GGT.566, as well as through the addition of Koi food, a typical component of the Culex larval laboratory diet. The modified diet was tested on both C. pipiens (Figure 3A) and C. quiquefasciatus (Figure 3C) larvae grown in scaled larval rearing trays. Under these conditions, the larvicides continued to induce significantly higher than expected C. pipiens male:female ratios (Figure 3A, $p<0.001$ ) resulting from significant female-specific mortality (Figure $3 \mathrm{~A}, p<0.001$ ); however, there was no significant impact on male $C$. pipiens survival (Figure $3 \mathrm{~A}, p>0.05$ ) or wing lengths (Figure $3 \mathrm{~B}, p>0.05$ ), which correlate with fitness. Similarly, the modified diet permitted scaled rearing of fit C. quinquefasciatus males, with GGT.566 diet treatments inducing significantly higher male:female ratios (Figure $3 C, p<0.001$ ) resulting from significant female-specific mortality (Figure $3 C, p<0.001$ ), but no significant impact on male $C$. quinquefasciatus survival (Figure $3 \mathrm{C}, p<0.001$ ) or fitness, which was not measured directly but estimated through measurement of wing lengths, which were not significantly different (Figure 3D, $p>0.05$ ). 

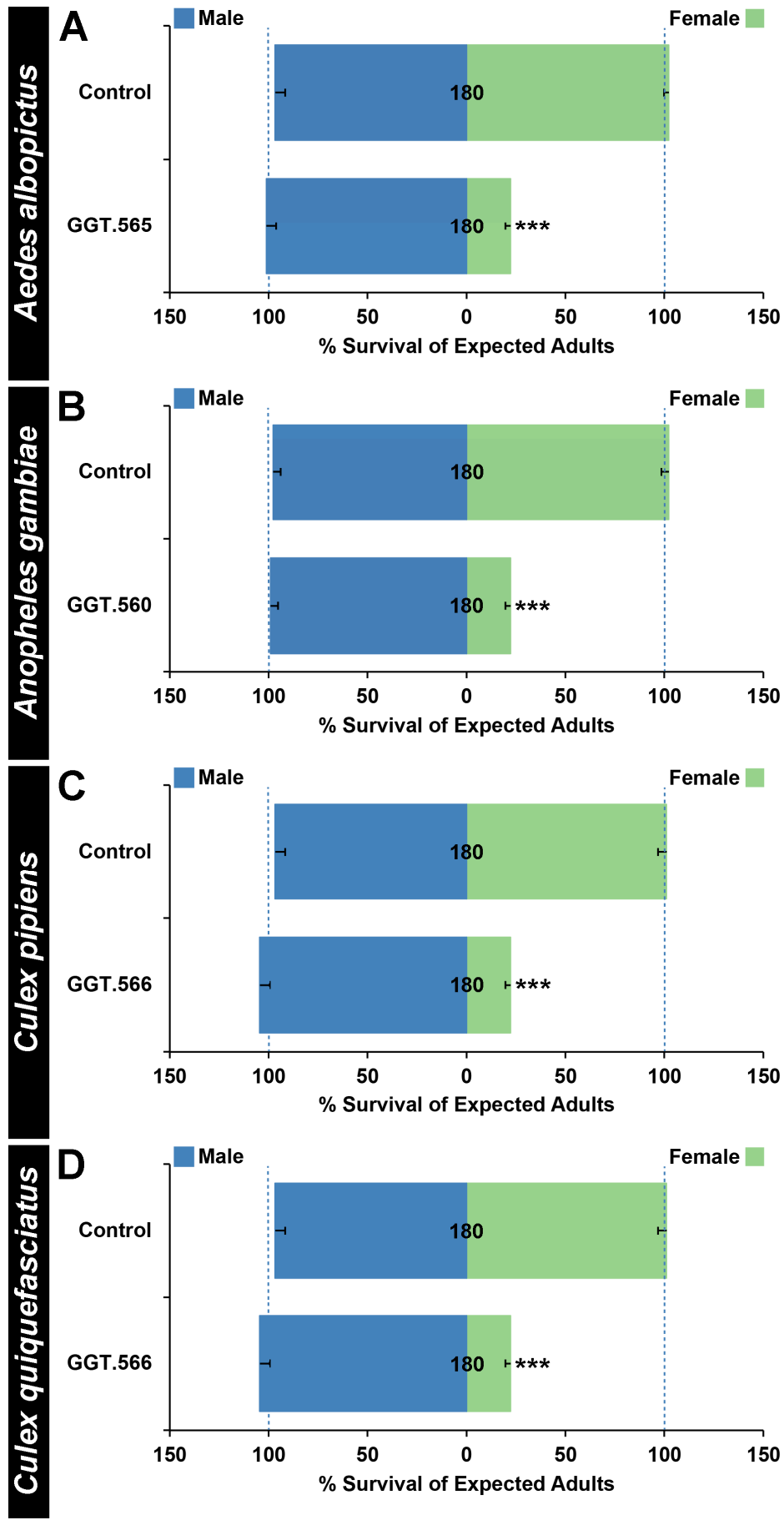

Figure 2. A female larval requirement for GGT function in multiple species of mosquitoes. Larval consumption of yeast-interfering RNA larvicides corresponding to the A. albopictus (GGT.566 in (A)), A. gambiae (GGT.560 in (B)), C. pipiens (GGT.566 in (C)), and C. quinquefasciatus (GGT.566 in (D)) GGT genes results in significant female-specific mortality in each species $\left({ }^{* * *}=p<0.001\right.$, chi-squared test), but does not impact male survival ((A-D), $p>0.05$, chi-squared test). Rearing larvae on controlinterfering RNA yeast, which lacks a known target in mosquitoes, had no significant impact on female or male survival ((A-D), $p>0.05$, chi-squared test). Data are displayed as mean survival through adulthood, with error bars corresponding to SEM. The N numbers corresponding to each treatment are indicated on the respective columns of each bar graph, and error bars represent standard errors of the mean. 

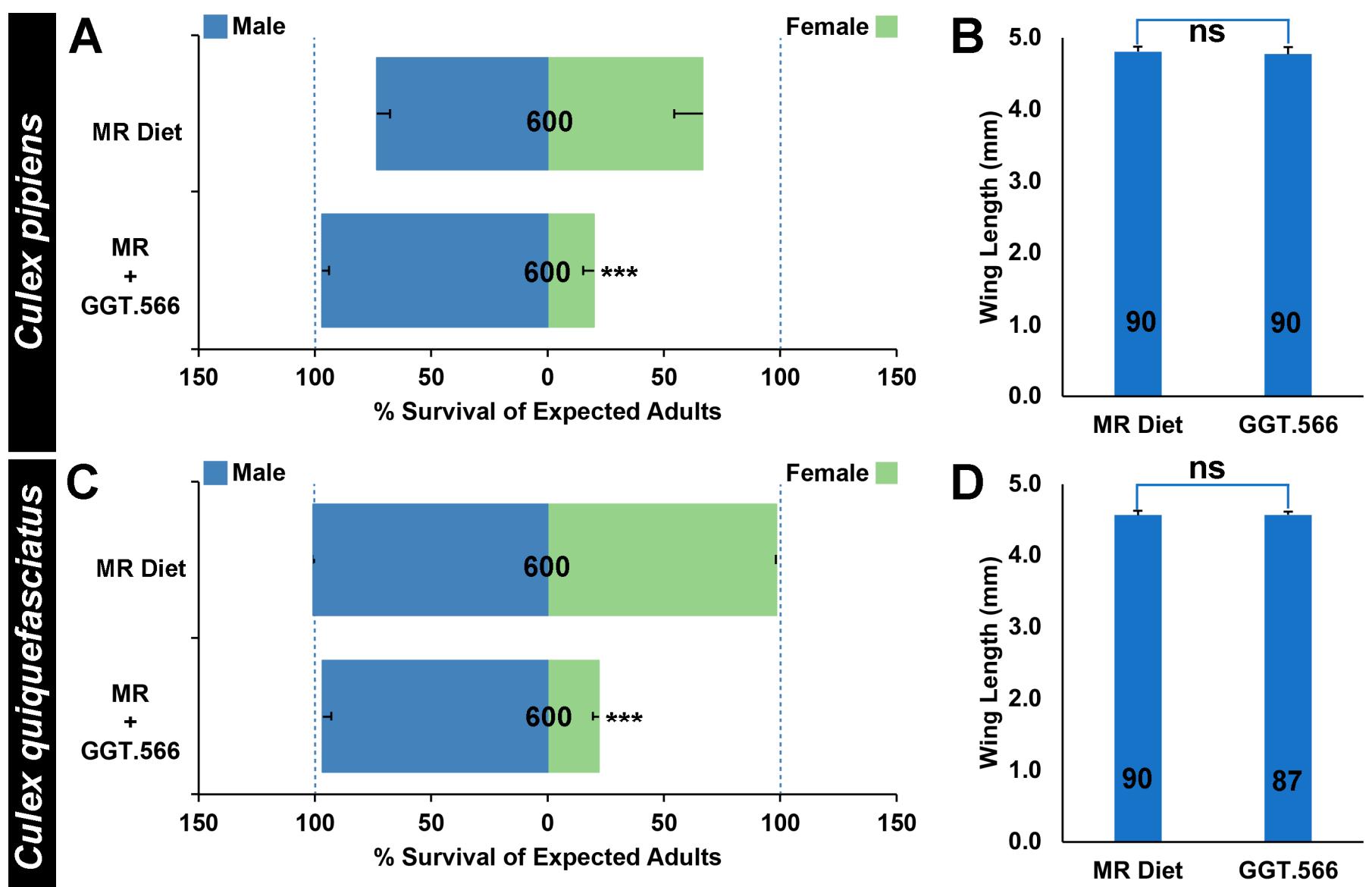

Figure 3. GGT.566 yeast facilitates scaled production of fit C. pipiens complex males. Incorporation of GGT.566 yeast into a Culex larval mass-rearing diet (MR+GGT.566) induced significant female C. pipiens ((A), ${ }^{* * *}=p<0.001$, chi-squared test) and C. quinquefasciatus $\left((\mathbf{C}),{ }^{* * *}=p<0.001\right.$, chi-squared test) mortality, yet did not significantly impact male survival $((\mathbf{A}, \mathbf{C}) p>0.05$, chi-squared test) or wing size (C. pipiens male wing results are shown in $(\mathbf{B})$ and $C$. quinquefasciatus male wing results are shown in (D); $p>0.05$ vs. mass-rearing diet results for both sets of wing size data in (C,D), Student's $t$-test). Data are displayed as mean survival through adulthood in $(\mathbf{A}, \mathbf{C})$ and mean adult male wing size in (B,D). Mosquitoes reared on the standard MR diet served as controls in all of these experiments (A-D). The $\mathrm{N}$ numbers corresponding to each treatment are indicated on the respective columns of each bar graph, and error bars represent standard errors of the mean. ns: no difference.

\section{Discussion}

\subsection{Sex Separation through Female-Specific Yeast RNA Larvicides}

RNAi-based larvicides [28,31] and adulticides [32,33] are presently being assessed and may one day be incorporated into integrated mosquito control programs. Here, we provide evidence that RNAi-based insecticides could also facilitate mosquito sex separation, a requirement for SIT, IIT, as well as several genetic-based mosquito control technologies that depend on the mass release of male mosquitoes. Papathanos et al. [34] recommend that sex-sorting methodology should be stable, simple, and lead to the isolation of competitive males, criteria that are fulfilled by the yeast system described here (Figures 1-3), which is also amenable to cost-effective and scalable production of shelf-stable yeast which can be dried and distributed for use in resource-limited regions of the world [28,31]. Moreover, yeast sex sorting systems, which are based on conditional gene silencing, induce no permanent changes to the mosquito genome, bypassing the need to transgenically manipulate existing mosquito strains that have been developed for population-based mosquito control strategies. Furthermore, the yeast would be heat-killed prior to use 
indoors at mass-rearing facilities, requiring no release of live GMOs into the environment. Thus, the GGT-silencing female-specific yeast-interfering RNA strains described herein,

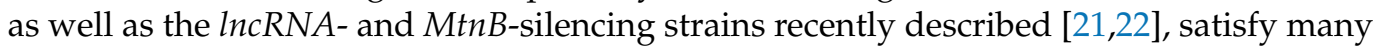
of the desired criteria for an effective sex-sorting system [34]. These findings suggest that future efforts to scale yeast production [28] for distribution to mass-rearing mosquito facilities will be useful.

Here, we demonstrated that replacement of the nutritional yeast component of an $A$. aegypti larval mass-rearing diet [30] with yeast that silences GGT resulted in the production of male mosquitoes at a five-fold higher number than females (Figure 1E). These results were similar to those described for yeast strains that silence $M t n B$ [22] or $\operatorname{lncRNA}$ [21] genes, except that the GGT.546 yeast strain induces female-specific death during the third instar, slightly earlier than the other strains, which killed fourth-instar larvae. Given that earlier death of females is expected to reduce rearing costs [34], use of the GGT.546 strain may offer an advantage over the other yeast strains [21,22]. Moreover, use of the GGT.560 strain for targeting A. gambiae (Figure 2B) yielded higher levels of female mortality than did the MtnB.523 yeast strain [22], (78 $\pm 3 \%$ vs. $43 \pm 5 \%$, respectively), suggesting that this strain may be a better choice for the separation of $A$. gambiae. The availability of multiple strains leads to the question of whether mixing yeast strains could lead to higher levels of female mortality. Preliminary data in which multiple types of yeast were simultaneously fed to the mosquito larvae suggest that this is not the case. However, a strain that produces high levels of multiple shRNAs has yet to be constructed, and engineering such a strain may prove useful.

Given that none of the strains generated to date have resulted in the elimination of all females (Figures 1-3; [21,22]), the present RNAi-based yeast systems will not be sufficient as a stand-alone strategy for mass male mosquito production. In addition to exploring methods of producing higher levels of one or more shRNAs, it could also be helpful to combine yeast technology with other existing sex separation methods that have been developed. A total of $>99 \%$ male separation serves as the present standard, and this standard has been achieved in A. aegypti through the use of a sophisticated camera system [8], as well as through fluorescent-based sorting of mosquito larvae using complex parametric analyzer and sorter (COPAS-) flow cytometry for sex separation in several species $[4,35,36]$. Although these systems are extremely effective, it is not yet clear if this automated technology can be adapted for use in all disease vector mosquito species or readily deployed worldwide, particularly to locations with limited resources. Aedes and Culex mosquitoes display sexually dimorphic size and growth rates, with female pupae developing more slowly and being larger in size, facilitating the early physical removal of small pupae. Likewise, pupae display differences in terminal abdominal segments that facilitate visual separation [5]. Replacing nutritional yeast with larvicidal yeast during the rearing process could serve to reduce the labor-intensive nature of manual separation strategies. Furthermore, combination of yeast larvicide-based sorting with behavioral sorting techniques could also prove useful. For example, females can be drawn to host cues, permitting separation [5]. Combining yeast larvicide use with these types of separation techniques could facilitate broadened use of emerging population-based mosquito control strategies.

\subsection{Scaled Rearing Culex pipiens Complex Males}

Culex pipiens complex mosquitoes, which transmit both arboviruses, such as West Nile virus, and parasites that cause lymphatic filariasis, pose significant threats to human health [1]. These mosquitoes also present severe threats to birds located on islands throughout the tropics, where avifauna are particularly susceptible to population declines and extinctions resulting from the introduction of non-native pathogens transmitted by Culex mosquitoes. The development of SIT, IIT, and transgenic-based strategies for populationbased Culex mosquito control on islands would be useful, but the development of such tools has been somewhat delayed with respect to comparable work in A. aegypti and A. gambiae. 
A relative lack of focus on Culex has resulted in the need for improved culturing methods, advances in genetic modification technology, Wolbachia transinfections, and further development and expansion of SIT programs, and as the development of population-based Culex control technologies progresses, the need for sex separation methodologies to facilitate these procedures is growing $[4,5]$. Female-specific yeast larvicide technology could thus particularly benefit the development and implementation of population-based control strategies for Culex (Figure 3), for which sex-sorting methods outside of visual and physical separation have yet to be established $[4,5]$.

Previous work demonstrated that silencing the C. quinquefasciatus MtnB gene resulted in female-specific larval death [22], but the target site for the yeast-interfering RNA larvicide used to target this gene was not conserved in C. pipiens. The GGT.566 target site is conserved in both species, and successful incorporation of yeast larvicide GGT.566 into a Culex rearing diet led to significant female mortality in both Culex species (Figure $3 \mathrm{~A}, \mathrm{C}$ ). It is possible that this site could be conserved in other species of Culex vector mosquitoes, which would permit extension of this technology to other vectors. This could be particularly beneficial to regions where it is anticipated that multiple species of Culex mosquitoes may be present, which may complicate population-based control efforts. Although $97 \%$ survival was observed in GGT.566-treated C. pipiens males, only $67 \%$ C. pipiens male survival was observed when larvae were reared on the unmodified standard mass-rearing diet used in this study (Figure 3A). It is likely that GGT.566-treated male larvae in larvicide-treated containers experienced less competition for food (due to female larval death) and also ate dead female larvae in addition to the food supplied. The eating of dead larvae, which we often observe in our yeast larvicide-treated containers, could perhaps serve to improve male larval nutrition and survival in rearing trays with high larval densities, a potential added benefit for mass-rearing facilities if yeast RNAi sex separation technology were to be employed. This could be further explored. Moreover, our results suggest that the Culex diet used in the present study could stand to be further optimized.

\subsection{Female-Specific Lethal Larvicides: Implications for Mosquito Sex Chromosome Evolution}

The findings reported herein could have implications for the study of sex chromosome evolution [37-39], which is believed to initiate with a homologous pair of autosomes that acquire sex-determining loci, eventually leading to a proto- $Y$ chromosome with a male fertility locus (M), as well as a proto- $X$ chromosome carrying a male sterility locus (m). Suppressed recombination in the sex-determining region develops and eventually spreads along the proto-sex chromosomes, which evolve into separate heteromorphic $\mathrm{X}$ and Y chromosomes. Unlike A. gambiae, which has evolved a Y chromosome with the male-determining Yob gene [29], Culicines have retained homomorphic sex chromosomes, though a sex-differentiated region of suppressed recombination is believed to extend approximately $100 \mathrm{Mb}$ beyond the sex determination M/m locus in A. aegypti [15,17,40-43]. Despite the different rates of sex chromosome evolution in mosquitoes, this investigation functionally verified that GGT is required for survival of female larvae in A. aegypti (Figure 1), A. albopictus (Figure 2A), A. gambiae (Figure 2B), C. pipiens (Figures 2C and 3A), and $C$. quinquefasciatus (Figures 2D and $3 \mathrm{C}$ ). These findings, combined with a recent study that identified a conserved female-specific larval requirement for $M t n B$ in mosquitoes [22], suggest that loci which cause sex-specific lethal effects that shape the boundaries of the sexdetermination locus had begun to accumulate on the sex chromosomes prior to separation of the Culicine and Anopheline lineages.

Krzywinska et al. [20] described sex-specific lethality in A. aegypti that was associated with the inheritance of sex chromosomes produced through male meiotic recombination events that had occurred between the sex-determination locus and a linked EGFP-positive transgene, which served as a marker. They posed several explanations for this, and one that is consistent with both the MtnB study [22], as well as the GGT silencing results presented here, suggested that such recombination events might result in loss of a haploinsufficient sex chromosome gene for which $\mathrm{m} / \mathrm{m}$ females require two copies. If GGT, as well as 
$M t n B$ [22], $\mathrm{M} / \mathrm{m}$ locus region $\operatorname{lnc} R N A$ genes [21], and perhaps other genes in the $\mathrm{M} / \mathrm{m}$ region are haploinsuffient in $A$. aegypti females, any female that inherits a recombinant $\mathrm{m}$ chromosome lacking a copy of any of these genes will die, a phenomenon that is sometimes referred to as 'suppression of recombination' due to the lack of resulting female offspring in the adult population. Thus, the early presence of haploinsufficient genes adjacent to the sex determination locus - prior to separation of the Culicines and Anophelines-may have helped to shape the boundaries of the sex determination region during mosquito sex chromosome evolution. Given these findings, it will be interesting to further assess this haploinsufficiency hypothesis and to assess the molecular mechanisms that might underlie it.

\section{Materials and Methods}

\subsection{Mosquito Strains and Rearing}

Mosquitoes were reared as described [22,44], except that an artificial membrane feeding system (Hemotek Limited, Blackburn, UK) was used to blood-feed adult females with sheep blood that had been purchased from a commercial vendor (HemoStat Laboratories, Dixon, CA, USA). The following strains of mosquitoes were used in these studies: A. aegypti Liverpool-IB12 strain, A. albopictus Gainesville strain (BEI Resources, NIAID, NIH: A. albopictus MRA-804, contributed by Sandra A. Allan), A. gambiae G3 strain (BEI Resources, NIAID, NIH: MRA-112, contributed by Mark Q. Benedict), and C. quinquefasciatus Strain JHB (provided by the Centers for Disease Control and Prevention for distribution by BEI Resources, NIAID, NIH: NR-43025). The C. pipiens strain was established from ovitrap collections in Niles, Michigan, with approximately 10th generation larvae used in these studies.

\subsection{Larval siRNA Soaking Experiments}

The Integrated DNA Technologies (IDT) Dicer-Substrate siRNA (DsiRNA) tool [45] was used to identify small interfering RNAs (siRNAs) corresponding to mosquito GGT genes (Table 1). These custom siRNAs (see Table 1), as well as a control siRNA with no known mosquito target [46], were purchased from IDT (Coralville, IA, USA) and used in larval soaking experiments. These assays, which were performed in duplicate as described [47], were executed using 20 first-instar larvae (L1) soaked in $0.5 \mu \mathrm{g} / \mu \mathrm{L}$ siRNA (Table 1) for $4 \mathrm{~h}$ and subsequently reared as described [26]. The chi-squared test was used to identify statistically significant differences between the observed and expected survival of adult female and male mosquitoes; a 1 female: 1 male ratio was expected on the basis of extensive control experiments performed in the insectary with the mosquito strains indicated above.

\subsection{Production of Yeast RNAi Larvicides}

RNAi yeast strains for silencing mosquito GGT genes were generated as described [27] by cloning of custom shRNA expression cassettes (synthesized by Invitrogen, Carlsbad, CA, USA) corresponding to GGT target gene sequences of interest (Table 1). The shRNA expression cassettes were inserted into the $p R S 426 G P D$ shuttle vector [48] and used to transform $S$. cerevisiae CEN.PK yeast (genotype MATa/a ura3-52/ura3-52 trp1-289/trp1-289 leu2-3_112/leu23_112 his3 $\Delta 1 /$ his3 $\triangle 1$ MAL2-8C/MAL2-8C SUC2/SUC2 [49]. Dried inactivated yeastinterfering RNA larvicide was prepared as previously detailed [50] following confirmation of shRNA expression via PCR amplification, which was performed as described [33] using the following primers: forward $5^{\prime}$-TGCCGTAAGCATGTTTGAATGCTTC-3' (specific to the $3^{\prime}$ side of the A. aegypti GGT.546 hairpin), forward 5'-GACGGTAAACTGGTCAAACAGATA G-3' (specific to the $3^{\prime}$ side of the A. gambiae GGT.560 hairpin), forward 5'-TAAAACTAAGTA GTATCCCACTTGC-3' (specific to the $3^{\prime}$ side of the A. albopictus GGT.565 hairpin), forward $5^{\prime}$-TACTGTTTTCATCAATCGTAAGTCT-3' (specific to the $3^{\prime}$ side of the $C$. quinquefasciatus GGT.566 hairpin), forward 5'-ACGCTAACATCTATCAGTGC-3' (specific to the $3^{\prime}$ side of the control hairpin, as previously described [33]) in combination with the reverse primer 
$5^{\prime}$-TCCTTCCTTTTCGGTTAGAGC-3' (specific to the terminator sequence of all five yeast strains, as described [33]). Figure S1 was labeled using Powerpoint software.

\subsection{Yeast Larvicide Assays}

The yeast larvicides were evaluated through assays that were performed as described [50] and which conformed to the WHO larvicide testing guidelines [51]. In these assays, 20 freshly hatched first-instar larvae were placed in $500 \mathrm{~mL}$ plastic cups with $50 \mathrm{~mL}$ of distilled water and $50 \mathrm{mg}$ of dried inactivated control-interfering RNA yeast [26] or dried inactivated yeast corresponding to GGT. The adult emergence rates and sexes of treated mosquitoes were recorded. Data compiled from four replicate trials were evaluated using the chi-squared test to identify statistically significant differences between the observed and expected 1 female: 1 male ratios, female survival, and male survival. The 1 female: 1 male ratio was established on the basis of extensive evaluation with control-interfering RNA yeast in each mosquito species evaluated.

\subsection{Verification of Target Gene Silencing}

Target gene silencing was verified through in situ hybridization assays that were executed on L4 larval brains as described [52]. Riboprobe corresponding to GGT (AAEL017331) was synthesized as specified [53] and used in these assays. Four biological replicate experiments were conducted on larvae (20 per replicate) that were treated with GGT yeast larvicides, as detailed above. After processing, larval brain tissue was mounted, viewed on a Zeiss Axioimager (Carl Zeiss Microscopy, LLC, Thornwood, NY, USA), and imaged using a Spot Flex camera (Diagnostic Instruments, Inc., Sterling Heights, MI, USA). Mean gray value analyses, which measure average signal intensity over the selected area, were conducted using FIJI ImageJ software [54], permitting the comparison of quantified digoxigenin-labeled transcript signals in the brains of larvicide treated vs. control larvae as described [55]. Data were statistically evaluated using Student's $t$-test.

\subsection{Scaled Rearing Trials}

For scaled rearing of $A$. aegypti, 500 larvae were placed in $34 \mathrm{~cm} \times 25 \mathrm{~cm} \times 4 \mathrm{~cm}$ trays (1426B, Bioquip, Rancho Dominquez, CA, USA), which contained $1 \mathrm{~L}$ of distilled water. A. aegypti larvae were fed with the Zhang et al. mass-rearing diet [30], which consisted of a slurry containing $150 \mathrm{mg}$ of ground shrimp (Tetra GMBH, Melle, Germany) and $250 \mathrm{mg}$ bovine liver powder (MP Biomedicals, Santa Ana, CA, USA) mixed with $10 \mathrm{~mL}$ water, with the nutritional yeast diet component [30] replaced with dried GGT.546 yeast for sex separation treatments; larvae reared on the unmodified Zhang et al. [30] standard mass-rearing diet prepared with nutritional yeast served as a control. The nutritional yeast (control) or GGT.546 larvicidal yeast-interfering RNA component was combined with the shrimp-liver powder slurry and provided to larvae as follows: $100 \mathrm{mg}$ at L1, L2; $200 \mathrm{mg}$ at L3 and L4. For scaled rearing of Culex larvae, C. quinquefasciatus and C. pipiens were reared on a diet consisting of Koi food (Doctors Foster and Smith Koi Food, Rhinelander, WI, USA) mixed with either nutritional yeast (control) or larvicidal GGT.566 yeast and combined with $10 \mathrm{~mL}$ of water that was provided to the larvae as a slurry. For rearing one tray of 200 larvae, the amounts of Koi food and yeast combined with water and fed during each larval instar were as follows: L1: $120 \mathrm{mg}$ Koi food + $40 \mathrm{mg}$ yeast; L2: $120 \mathrm{mg}$ Koi food $+40 \mathrm{mg}$ yeast; L3: 160 mg Koi Food + 160 mg yeast; L4: 160 mg Koi Food + 160 mg yeast.

For all species, the larval rearing trays were examined daily for pupae, which were subsequently removed, sorted by sex, and counted. Mortality and sex ratio data for each control or larvicide treatment were evaluated in three replicate trials. Statistically significant differences between the observed and expected 1 male: 1 female survival ratios were evaluated using the chi-squared test, and the fitness of surviving adult males was estimated through comparisons of wing lengths, which correlate with fitness and were measured as described [55] and evaluated with Student's t-test. 


\section{Conclusions}

This investigation has uncovered a conserved female-specific requirement for GGT in distantly related disease vector mosquitoes. The results in conjunction with other recent functional analyses of genes in the sex-determining region of $A$. aegypti [21,22], as well as the orthologs of these genes in other species of mosquitoes [22], may have important implications for the study of mosquito sex chromosome evolution. Moreover, femalespecific yeast-interfering RNA technologies that exploit the conserved female-specific requirement for GGT, as well as other $\mathrm{M} / \mathrm{m}$ locus region genes and their orthologs in other mosquitoes, could support production of adult males. The use of other microbial systems could also be trialed for sex separation in Culex larvae. For example, Pichia pastoris [56] and Escherichia coli $[57,58]$, which have also proven useful for expression and delivery of interfering RNA to mosquito larvae, could likely be used for silencing mosquito GGT genes and can be further assessed in C. pipiens complex mosquitoes. Moreover, given that the use of microbial systems for sex separation would require large-scale microbe production, it would be useful to pilot and optimize the scaled production of different microbes with the goal of determining which microbial-interfering RNA expression systems are the most economical and suited for scaled production. Such advancements would facilitate emerging population-based mosquito control strategies, particularly for species of the C. pipiens complex and other relatively less well-characterized mosquito species for which sex separation technologies are lacking.

Supplementary Materials: The following is available online at https:/ /www.mdpi.com/article/10 .3390/pathogens11020169/s1, Figure S1: Expression of control, GGT.560, GGT.565, and GGT.566 shRNA was confirmed through PCR reactions, which produced $100 \mathrm{bp}$ amplicons from cDNA template prepared from total RNA isolated from each corresponding yeast strain. Results from two separate cDNA preparations (labeled 1 and 2 for each strain) are shown. No bands were produced in reactions that lacked cDNA template, which are marked as (-). The GGT.560, GGT.565, and GGT.566 primer sets failed to amplify bands from the control-interfering RNA yeast cDNA preparations (labeled cntrl 1 and 2). A DNA standard ladder is included in the far left and far right lanes.

Author Contributions: Conceptualization: M.D.-S.; methodology: K.M., L.S., P.L. and M.D.-S.; formal analysis: K.M., P.L., J.B.R. and M.D.-S.; investigation: K.M., L.S., P.L., J.B.R., J.K.M., J.K. and J.I.; writing-original draft preparation: K.M. and M.D.-S.; writing-review and editing: K.M., J.B.R. and M.D.-S.; supervision: K.M. and M.D.-S.; project administration: M.D.-S.; funding acquisition: M.D.-S. All authors have read and agreed to the published version of the manuscript.

Funding: This investigation was funded through NIH-NIAID grant awards 1 R21 AI144256-01 and R21AI156170-01 to M.D.-S. NIH-NIAID was not involved in study design, data collection and interpretation, or the decision to submit the work for publication.

Institutional Review Board Statement: Not applicable.

Informed Consent Statement: Not applicable.

Data Availability Statement: All data are provided within the text.

Acknowledgments: We thank Na Wei and Chien-Wei Wang for advice on yeast strain production. We thank past and present members of the lab for useful discussions.

Conflicts of Interest: MDS is an inventor on U.S. patent application number 62/751,052. The pending patent application did not influence her data interpretation or decision to publish this work. All other authors declare that they have no competing interests.

\section{References}

1. World Health Organization. Vector-Borne Diseases. Available online: https://www.who.int/news-room/fact-sheets/detail/ vector-borne-diseases (accessed on 8 December 2021).

2. Environmental Protection Agency. Joint Statement on Mosquito Control in the United States. Available online: https://www.epa. gov/mosquitocontrol/joint-statement-mosquito-control-united-states (accessed on 8 December 2021).

3. Knipling, E. Possibilities of insect control or eradication through the use of sexually sterile males. J. Econ. Entomol. 1955, 48, 459-462. [CrossRef] 
4. Lutrat, C.; Giesbrecht, D.; Marois, E.; Whyard, S.; Baldet, T.; Bouyer, J. Sex Sorting for Pest Control: It's Raining Men! Trends Parasitol. 2019, 35, 649-662. [CrossRef] [PubMed]

5. Papathanos, P.A.; Bourtzis, K.; Tripet, F.; Bossin, H.; Virginio, J.F.; Capurro, M.L.; Pedrosa, M.C.; Guindo, A.; Sylla, L.; Coulibaly, M.B.; et al. A perspective on the need and current status of efficient sex separation methods for mosquito genetic control. Parasit. Vectors 2018, 11, 654. [CrossRef] [PubMed]

6. Zheng, X.; Zhang, D.; Li, Y.; Yang, C.; Wu, Y.; Liang, X.; Liang, Y.; Pan, X.; Hu, L.; Sun, Q.; et al. Incompatible and sterile insect techniques combined eliminate mosquitoes. Nature 2019, 572, 56-61. [CrossRef] [PubMed]

7. Kittayapong, P.; Ninphanomchai, S.; Limohpasmanee, W.; Chansang, C.; Chansang, U.; Mongkalangoon, P. Combined sterile insect technique and incompatible insect technique: The first proof-of-concept to suppress Aedes aegypti vector populations in semi-rural settings in Thailand. PLoS Negl. Trop. Dis. 2019, 13, e0007771. [CrossRef]

8. Crawford, J.E.; Clarke, D.W.; Criswell, V.; Desnoyer, M.; Cornel, D.; Deegan, B.; Gong, K.; Hopkins, K.C.; Howell, P.; Hyde, J.S.; et al. Efficient production of male Wolbachia-infected Aedes aegypti mosquitoes enables large-scale suppression of wild populations. Nat. Biotechnol. 2020, 38, 482-492. [CrossRef]

9. Carvalho, D.O.; McKemey, A.R.; Garziera, L.; Lacroix, R.; Donnelly, C.A.; Alphey, L.; Malavasi, A.; Capurro, M.L. Suppression of a Field Population of Aedes aegypti in Brazil by Sustained Release of Transgenic Male Mosquitoes. PLoS Negl. Trop. Dis. 2015, 9, e0003864. [CrossRef]

10. Wise de Valdez, M.R.; Nimmo, D.; Betz, J.; Gong, H.F.; James, A.A.; Alphey, L.; Black, W.C. Genetic elimination of dengue vector mosquitoes. Proc. Natl. Acad. Sci. USA 2011, 108, 4772-4775. [CrossRef]

11. Macias, V.M.; Ohm, J.R.; Rasgon, J.L. Gene Drive for Mosquito Control: Where Did It Come from and Where Are We Headed? Int. J. Environ. Res. Public Health 2017, 14, 1006. [CrossRef]

12. Adelman, Z.N.; Tu, Z. Control of Mosquito-Borne Infectious Diseases: Sex and Gene Drive. Trends Parasitol. 2016, 32, 219-229. [CrossRef]

13. McClelland, G.A.H. Sex-linkage in Aedes aegypti. Trans. R. Soc. Trop. Med. Hyg. 1962, 56, 4.

14. Newton, M.E.; Wood, R.J.; Southern, D.I. Cytological mapping of the M and D loci in the mosquito, Aedes aegypti (L.). Genetica 1978, 48, 137-143. [CrossRef]

15. Hall, A.B.; Basu, S.; Jiang, X.; Qi, Y.; Timoshevskiy, V.A.; Biedler, J.K.; Sharakhova, M.V.; Elahi, R.; Anderson, M.A.; Chen, X.G.; et al. A male-determining factor in the mosquito Aedes aegypti. Science 2015, 348, 1268-1270. [CrossRef] [PubMed]

16. Motara, M.A.; Rai, K.S. Giemsa C-banding patterns in Aedes (Stegomyia) mosquitoes. Chromosoma 1978, 70, 51-58. [CrossRef]

17. Matthews, B.J.; Dudchenko, O.; Kingan, S.B.; Koren, S.; Antoshechkin, I.; Crawford, J.E.; Glassford, W.J.; Herre, M.; Redmond, S.N.; Rose, N.H.; et al. Improved reference genome of Aedes aegypti informs arbovirus vector control. Nature 2018, 563, 501-507. [CrossRef]

18. Giraldo-Calderon, G.I.; Emrich, S.J.; MacCallum, R.M.; Maslen, G.; Dialynas, E.; Topalis, P.; Ho, N.; Gesing, S.; VectorBase, C.; Madey, G.; et al. VectorBase: An updated bioinformatics resource for invertebrate vectors and other organisms related with human diseases. Nucleic Acids Res. 2015, 43, D707-D713. [CrossRef]

19. Wood, R.J. Lethal genes on the sex chromosomes concealed in a population of the mosquito Aedes aegypti L. Genetica 1990, 46, 49-66. [CrossRef]

20. Krzywinska, E.; Kokoza, V.; Morris, M.; de la Casa-Esperon, E.; Raikhel, A.S.; Krzywinski, J. The sex locus is tightly linked to factors conferring sex-specific lethal effects in the mosquito Aedes aegypti. Heredity 2016, 117, 408-416. [CrossRef]

21. Mysore, K.; Hapairai, L.K.; Li, P.; Roethele, J.B.; Sun, L.; Igiede, J.; Misenti, J.K.; Duman-Scheel, M. A functional requirement for sex-determination M/m locus region lncRNA genes in Aedes aegypti female larvae. Sci. Rep. 2021, 11, 10657. [CrossRef]

22. Mysore, K.; Sun, L.; Roethele, J.B.; Li, P.; Igiede, J.; Misenti, J.K.; Duman-Scheel, M. A conserved female-specific larval requirement for $M t n B$ function facilitates sex separation in multiple species of disease vector mosquitoes. Parasit. Vectors 2021, 14, 338. [CrossRef]

23. Sinkins, S.P.; Gould, F. Gene drive systems for insect disease vectors. Nat. Rev. Genet. 2006, 7, 427-435. [CrossRef]

24. Larkin, A.; Marygold, S.J.; Antonazzo, G.; Attrill, H.; Dos Santos, G.; Garapati, P.V.; Goodman, J.L.; Gramates, L.S.; Millburn, G.; Strelets, V.B.; et al. FlyBase: Updates to the Drosophila melanogaster knowledge base. Nucleic Acids Res. 2021, 49, D899-D907. [CrossRef] [PubMed]

25. GARD, N. Glutathionuria. Available online: https:/ / rarediseases.info.nih.gov/diseases/10099/glutathionuria (accessed on 5 August 2021).

26. Hapairai, L.K.; Mysore, K.; Chen, Y.; Harper, E.I.; Scheel, M.P.; Lesnik, A.M.; Sun, L.; Severson, D.W.; Wei, N.; Duman-Scheel, M. Lure-and-Kill Yeast Interfering RNA Larvicides Targeting Neural Genes in the Human Disease Vector Mosquito Aedes aegypti. Sci. Rep. 2017, 7, 13223. [CrossRef] [PubMed]

27. Mysore, K.; Hapairai, L.K.; Sun, L.; Harper, E.I.; Chen, Y.; Eggleson, K.K.; Realey, J.S.; Scheel, N.D.; Severson, D.W.; Wei, N.; et al. Yeast interfering RNA larvicides targeting neural genes induce high rates of Anopheles larval mortality. Malar. J. 2017, 16, 461. [CrossRef]

28. Duman-Scheel, M. Saccharomyces cerevisiae (Baker's Yeast) as an Interfering RNA Expression and Delivery System. Curr. Drug Targets 2019, 20, 942-952. [CrossRef] [PubMed]

29. Krzywinska, E.; Krzywinski, J. Effects of stable ectopic expression of the primary sex determination gene Yob in the mosquito Anopheles gambiae. Parasit. Vectors 2018, 11, 648. [CrossRef] [PubMed] 
30. Zhang, D.; Zhang, M.; Wu, Y.; Gilles, J.R.L.; Yamada, H.; Wu, Z.; Xi, Z.; Zheng, X. Establishment of a medium-scale mosquito facility: Optimization of the larval mass-rearing unit for Aedes albopictus (Diptera: Culicidae). Parasit. Vectors 2017, 10, 569. [CrossRef]

31. Wiltshire, R.M.; Duman-Scheel, M. Advances in oral RNAi for disease vector mosquito research and control. Curr. Opin. Insect Sci. 2020, 40, 18-23. [CrossRef]

32. Hapairai, L.K.; Mysore, K.; Sun, L.; Li, P.; Wang, C.W.; Scheel, N.D.; Lesnik, A.; Scheel, M.P.; Igiede, J.; Wei, N.; et al. Characterization of an adulticidal and larvicidal interfering RNA pesticide that targets a conserved sequence in mosquito $G$ protein-coupled dopamine 1 receptor genes. Insect Biochem. Mol. Biol. 2020, 120, 103359. [CrossRef]

33. Mysore, K.; Hapairai, L.K.; Sun, L.; Li, P.; Wang, C.W.; Scheel, N.D.; Lesnik, A.; Igiede, J.; Scheel, M.P.; Wei, N.; et al. Characterization of a dual-action adulticidal and larvicidal interfering RNA pesticide targeting the Shaker gene of multiple disease vector mosquitoes. PLoS Negl. Trop. Dis. 2020, 14, e0008479. [CrossRef]

34. Papathanos, P.A.; Bossin, H.C.; Benedict, M.Q.; Catteruccia, F.; Malcolm, C.A.; Alphey, L.; Crisanti, A. Sex separation strategies: Past experience and new approaches. Malar. J. 2009, 8 (Suppl. 2), S5. [CrossRef] [PubMed]

35. Catteruccia, F.; Benton, J.P.; Crisanti, A. An Anopheles transgenic sexing strain for vector control. Nat. Biotechnol. 2005, 23, 1414-1417. [CrossRef] [PubMed]

36. Bernardini, F.; Galizi, R.; Menichelli, M.; Papathanos, P.A.; Dritsou, V.; Marois, E.; Crisanti, A.; Windbichler, N. Site-specific genetic engineering of the Anopheles gambiae Y chromosome. Proc. Natl. Acad. Sci. USA 2014, 111, 7600-7605. [CrossRef] [PubMed]

37. Charlesworth, B. Model for evolution of Y chromosomes and dosage compensation. Proc. Natl. Acad. Sci. USA 1978, 75, 5618-5622. [CrossRef]

38. Charlesworth, B. The evolution of sex chromosomes. Science 1991, 251, 1030-1033. [CrossRef]

39. Charlesworth, D.; Charlesworth, B.; Marais, G. Steps in the evolution of heteromorphic sex chromosomes. Heredity 2005, 95, 118-128. [CrossRef]

40. Juneja, P.; Osei-Poku, J.; Ho, Y.S.; Ariani, C.V.; Palmer, W.J.; Pain, A.; Jiggins, F.M. Assembly of the genome of the disease vector Aedes aegypti onto a genetic linkage map allows mapping of genes affecting disease transmission. PLoS Negl. Trop. Dis. 2014, 8, e2652. [CrossRef]

41. Fontaine, A.; Filipovic, I.; Fansiri, T.; Hoffmann, A.A.; Cheng, C.; Kirkpatrick, M.; Rasic, G.; Lambrechts, L. Extensive Genetic Differentiation between Homomorphic Sex Chromosomes in the Mosquito Vector, Aedes aegypti. Genome Biol. Evol. 2017, 9, 2322-2335. [CrossRef]

42. Miller, J.R.; Koren, S.; Dilley, K.A.; Puri, V.; Brown, D.M.; Harkins, D.M.; Thibaud-Nissen, F.; Rosen, B.; Chen, X.G.; Tu, Z.; et al. Analysis of the Aedes albopictus C6/36 genome provides insight into cell line utility for viral propagation. Gigascience 2018, 7, gix135. [CrossRef]

43. Gomulski, L.M.; Mariconti, M.; Di Cosimo, A.; Scolari, F.; Manni, M.; Savini, G.; Malacrida, A.R.; Gasperi, G. The Nix locus on the male-specific homologue of chromosome 1 in Aedes albopictus is a strong candidate for a male-determining factor. Parasit. Vectors 2018, 11, 647. [CrossRef]

44. Clemons, A.; Mori, A.; Haugen, M.; Severson, D.W.; Duman-Scheel, M. Culturing and egg collection of Aedes aegypti. Cold Spring Harb. Protoc. 2010, 2010, pdb prot5507. [CrossRef]

45. IDT. Custom Dicer-Substrate siRNA (DsiRNA). Available online: https://www.idtdna.com/site/order/designtool/index/ DSIRNA_CUSTOM (accessed on 16 April 2017).

46. Tomchaney, M.; Mysore, K.; Sun, L.; Li, P.; Emrich, S.J.; Severson, D.W.; Duman-Scheel, M. Examination of the genetic basis for sexual dimorphism in the Aedes aegypti (dengue vector mosquito) pupal brain. Biol. Sex Differ. 2014, 5, 10. [CrossRef] [PubMed]

47. Singh, A.D.; Wong, S.; Ryan, C.P.; Whyard, S. Oral delivery of double-stranded RNA in larvae of the yellow fever mosquito, Aedes aegypti: Implications for pest mosquito control. J. Insect. Sci. 2013, 13, 69. [CrossRef] [PubMed]

48. Mumberg, D.; Muller, R.; Funk, M. Yeast vectors for the controlled expression of heterologous proteins in different genetic backgrounds. Gene 1995, 156, 119-122. [CrossRef]

49. Van Dijken, J.P.; Bauer, J.; Brambilla, L.; Duboc, P.; Francois, J.M.; Gancedo, C.; Giuseppin, M.L.; Heijnen, J.J.; Hoare, M.; Lange, H.C.; et al. An interlaboratory comparison of physiological and genetic properties of four Saccharomyces cerevisiae strains. Enzym. Microb. Technol. 2000, 26, 706-714. [CrossRef]

50. Mysore, K.; Hapairai, L.K.; Wei, N.; Realey, J.S.; Scheel, N.D.; Severson, D.W.; Duman-Scheel, M. Preparation and Use of a Yeast shRNA Delivery System for Gene Silencing in Mosquito Larvae. Methods Mol. Biol. 2019, 1858, 213-231. [CrossRef]

51. WHO. Guidelines for Laboratory and Field Testing of Mosquito Larvicides; World Health Organization: Geneva, Switzerland, 2005.

52. Haugen, M.; Tomchaney, M.; Kast, K.; Flannery, E.; Clemons, A.; Jacowski, C.; Simanton Holland, W.; Le, C.; Severson, D.; Duman-Scheel, M. Whole-mount in situ hybridization for analysis of gene expression during Aedes aegypti development. Cold Spring Harb. Protoc. 2010, 2010, pdb prot5509. [CrossRef]

53. Patel, N.H. In Situ Hybridization to Whole Mount Drosophila Embryos. In A Laboratory Guide to RNA: Isolation, Analysis, and Synthesis; Krieg, P.A., Ed.; Wiley-Liss: New York, NY, USA, 1996; pp. 357-370.

54. Schindelin, J.; Arganda-Carreras, I.; Frise, E.; Kaynig, V.; Longair, M.; Pietzsch, T.; Preibisch, S.; Rueden, C.; Saalfeld, S.; Schmid, B.; et al. Fiji: An open-source platform for biological-image analysis. Nat. Methods 2019, 9, 676-682. [CrossRef] 
55. Mysore, K.; Sun, L.; Tomchaney, M.; Sullivan, G.; Adams, H.; Piscoya, A.S.; Severson, D.W.; Syed, Z.; Duman-Scheel, M. siRNAMediated Silencing of doublesex during Female Development of the Dengue Vector Mosquito Aedes aegypti. PLoS Negl. Trop. Dis. 2015, 9, e0004213. [CrossRef]

56. Van Ekert, E.; Powell, C.A.; Shatters, R.G., Jr.; Borovsky, D. Control of larval and egg development in Aedes aegypti with RNA interference against juvenile hormone acid methyl transferase. J. Insect. Physiol. 2014, 70, 143-150. [CrossRef]

57. Whyard, S.; Erdelyan, C.N.G.; Partridge, A.L.; Singh, A.D.; Beebe, N.W.; Capina, R. Silencing the buzz: A new approach to population suppression of mosquitoes by feeding larvae double-stranded RNAs. Parasit. Vectors 2015, 8, 96. [CrossRef] [PubMed]

58. Mabel, L.; Taracena, M.L.; Hunt, C.M.; Benedict, M.Q.; Pennington, P.M.; Dotson, E.M. Downregulation of female doublesex expression by oral-mediated RNA interference reduces number and fitness of Anopheles gambiae adult females. Parasit. Vectors 2019, 12, 170. [CrossRef] 